Ann. Génét. Sél. anim., 1976, 8 (I), 51-70.

REVIEW

\title{
LE SYNDROME D'ARTHROGRYPOSE ET DE PALATOSCHISIS (SAP) EN RACE BOVINE CHAROLAISE
}

\author{
BIBLIOGRAPHIE ANNOTÉE : I967-1975
}

\author{
J.-J. LAUVERGNE et A. FAUCON*(1) \\ Département de Génétique animale, \\ Centre national de Recherches zootechniques, I. N.R. A., \\ 78350 Jouy en Josas \\ * Institut technique de l'Élevage Bovin, \\ 149, rue de Bercy. \\ 75579 Paris Cedex 12
}

\section{RÉSUMÉ}

Pour la période allant de 1967 à 1975 on a trouvé 49 références sous la plume de 33 auteurs différents concernant le Syndrome d'Arthrogrypose et de Palatoschisis dans la race Charolaise (SAP). Lne bonne partie de ces sources (37 p. 100) est d'un type difficilement compilable par les méthodes documentaires habituelles car il s'agit de rapports à diffusion restreinte ou de compte rendus de réunions de groupe à existence plus ou moins formelle et plus ou moins brève. Les articles viennent principalement du Canada (34 p. 10o) et de la France (42 p. 10o) où des études ont commencé pratiquement en même temps, en 1966, sous la forme de collaboration entre unités de recherches et organismes techniques. Les résultats se recoupent et se complètent assez bien d'un pays à l'autre pour qu'aucun doute ne subsiste sur le caractère monofactoriel autosomal récessif du syndrome SAP propre à la race Charolaise et bien caractéristique. La divergence quant à la valeur de la pénétrance, faible en race pure (en France) et quasi totale chez les anormaux ayant au plus $7 / 8$ de sang Charolais au Canada est certaine mais demande des études plus précises. La fréquence du gène dans le Charolais en France est d'environ o,20. Ces deux dernières années on note un début de concertation internationale dans l'organisation des recherches alors que, dans le passé l'échange d'information a pu laisser à désirer.

\section{INTRODUCTION}

L'étude de certaines anomalies héréditaires des bovins domestiques peut se prolonger sur plusieurs décades, voire sur plus d'un demi-siècle, avant d'aboutir.

Par exemple, les premières études sur le culard (hypertrophie musculaire) qui existe dans

(1) Actuel coordinateur du Comité Technique de la F.I.A.E.R.B.C. 
plusieurs races, sur l'hypoplasie des canaux de Müller associée aux couleurs rouanne et blanche du Shorthorn ou sur l'hypoplasie des gonades également associée à un blanchissement en race des Montagnes suédoises, remontent aux tous débuts du siècle (LAUvergne, r968).

La cause de ces longs étalements semble résider dans le caractère ambigu du comportement de certains mutants qui ont des aspects favorables à côté d'autres qui le sont moins. La sélection artificielle viendra alors contrebalancer la sélection naturelle (ou vice versa) pour parfois aboutir à un maintien du facteur dans la population. On note souvent des expressivités variables et des pénétrances faibles qui compliquent aussi bien l'étude que l'éradication (LAUVERGNE, 1972).

Les publications consacrées à une anomalie existant dans plusieurs pays, peuvent alors être fort nombreuses et fort dispersées. Le rassemblement de la bibliographie se révèle dans ce cas comme une tâche nécessaire, à mener indépendamment des travaux de recherche proprement dits.

Reprenant un genre lancé par les éditeurs d'Animal Breeding Abstracts nous avions ainsi dressé, il y a quelques années, une bibliographie annotée du phénomène culard chez les bovins (LAUVERGNE et al., 1968).

Nous voudrions, dans le présent article nous pencher de la même façon sur une autre anomalie congénitale des bovins Charolais qui, elle aussi, semble présenter les caractéristiques d'une anomalie à étude longue. Il s'agit du syndrome, qu'en anticipant un peu nous nommerons SAP (Syndrome d'Arthrogrypose et de Palatoschisis). A première vue héréditaire monofactoriel ce syndrome a des localisations multiples (palais, membres, muscles, ...) et semble, nonobstant, se maintenir assez bien dans les populations. L'importance pratique de SAP est telle que le Comité Technique de la F.I.A.F.R.B.C. (1) s'emploie depuis quelques années à coordonner les études qui le concernent.

\section{MATÉRIEL ET MÉTHODES}

Il y a quatre principales sources de références pour SAP : les travaux français, canadiens et américains auxquels s'ajoutent les textes des délibérations des organes internationaux de la race qui ont été amené à traiter de l'anomalie.

La collecte des références françaises et internationales était relativement facile. Pour les articles d'outre-Atlantique on s'est appuyé sur les bibliographies des auteurs que l'on a complétées lors de contacts pris à Calgary et à Saskatoon en juillet 1975 .

Les références sont présentées alphabétiquement année par année. On a respecté la référenciation originale des articles sauf ajout éventuel de la date, quitte à combler les possibles lacunes dans la rubrique N.D.L.R. (note de la rédaction) placée en fin d'analyse. Dans cette même rubrique le lecteur trouvera parfois des commentaires qui se veulent plus subjectifs que ceux qui figurent dans l'analyse.

La bibliographie annotée proprement dite est, enfin, assortie de trois annexes. L'une donne la liste des abréviations contenues dans le texte. La seconde est un index des auteurs cités, avec leurs qualités et adresses. La troisième est une liste des références classées alphabétiquement par noms d'auteurs.

\section{BIBLIOGRAPHIE ANNOTÉE}

$$
1967
$$

[I] Lauvergne J.-J., Blin P. C.,, I967. Fissure palatine héréditaire associée à l'ankylose des membres dans la race Charolaise. Ann. Zootech., 16, 29I-300.

A partir de la dissection d'un veau recueilli en juin r 966 et portant de multiples anomalies (au palais et aux membres), on analyse les cas similaires déjà repérés dans deux élevages privés et dans les zones des deux centres d'insémination français de l'Allier et de la Saône-et-Loire. En admettant qu'il s'agisse d'une tare monofactorielle particulière à la race Charolaise, on hésite entre un comportement récessif et un comportement dominant à pénétrance incomplète.

(1) F.I.A.F.R.B.C. : Fédération internationale des Associations d'Éleveurs de la Race Bovine Charolaise. 
[2] Lauvergie J.-J., I967. Brève analyse des premiers résultats de l'enquête sur la fissure palatine en race Charolaise au C.I.A. de l'Allier. pp. $6+6$, ronéoté.

Action concertée entre le Département de Génétique animale de l'I.N.R.A. et le Centre Technique de Contrôle de la Descendance (C.T.C.D. : une section, dirigée par J.-M. DuPLAN, de l'Institut Technique de l'Élevage Bovin I.T.E.B., Paris) l'enquête portait sur la descendance de 24 jeunes taureaux Charolais mis à l'épreuve pour les qualités bouchères de leur descendance en race pure. Les veaux sont tous nés dans le département de l'Allier, zone d'action du centre d'insémination de l'Allier, au début de l'année 1967.

L'analyse a porté sur des données quasi complètes mais pas encore toutes vérifiées. On relevait alors 37 cas sûrs d'anormaux atteints au palais et aux membres et I I cas probables. Treize taureaux sur les 24 étaient transmetteurs apparents de la tare. Dans une hypothèse de récessivité simple à pénétrance complète en panmixie la fréquence des taureaux transmetteurs (c'est-à-dire hétérozygotes) semblait un peu faible.

N.D.L.R. : Il s'agit d'un rapport diffusé par le Département de Génétique de l'I.N.R.A., C.N.R.Z., Jouy en Josas. Le raisonnement de génétique de population admettait, en sous entendu, que la pénétrance était complète chez les homozygotes et que le gène avait diffusé également dans toute la race, atteignant partout une fréquence d'équilibre. Dans ces conditions on constate un assez grand écart entre les deux estimations de la fréquence génique d'une part par le comptage des anormaux $(q=0,127)$ et, de l'autre part par le comptage des mâles hétérozygotes détectés. Cette dernière estimation doit être en effet de 0,25 et non pas de 0,176 comme on l'avait faussement calculé à l'époque. Cette divergence, qui se répétera les années suivantes, conduira à élaborer l'hypothèse, plus tard vérifiée, de pénétrance incomplète.

\section{I968}

[3] Greeley R. G., Boyd C. L., Jolly D. G., 1968. Bovine anomalies and spastic paresis. Southwest. Vet., 12, 277-280.

Quatre veaux (deux Charolais purs et deux croisés) ont été examinés à la suite du veau décrit en 4. Les grassets étaient soudés en flexion. Les articulations du jarret et du boulet arrière étaient fléchies mais pouvaient être manœuvrées à la main. Chez trois de ces veaux les boulets avant étaient semblables aux boulets arrière. Deux d'entre eux avaient le palais fendu. Ils étaient tous actifs dans les limites de leur infirmité.

A la dissection après euthanasie, tous présentaient des anomalies osseuses, tendineuses, musculaires et nerveuses fort semblables à celles rapportées en 4. Les cinq veaux anomaliques étaient d'ailleurs apparentés. Les auteurs pensent qu'il s'agit de cas extrêmes de paralysie spasmodique présentant quelques variations d'expressivité.

N.D.L.R. : D'après ce que l'on sait maintenant - et confirmant ce que pensaient déjà hiefFer et CARTwRight en I970 [12] - on peut penser que les auteurs du Texas ont décrit, plutôt que des cas de parésie spasmodique, des cas de l'anomalie faisant l'objet de la présente bibliographique.

Thi Greeley R. G., Jolly D. G., 1968. A clue to the etiology of spastic paresis. Southwest. Vet., 12, r 89-191.

La dissection du train arrière paralysé d'un veau Charolais de 9 semaines réalisée au College of Veterinary Medicine de la Texas $A$ and $M$ University à College Station, Texas, met en évidence une luxation de la patella, une hypoplasie de la trochlée fémorale, l'absence d'un tendon, l'existence d'une branche aberrante du nerf sciatique et le raccourcissement des muscles postérieurs de la cuisse. Les défauts tendineux et osseux rendent compte de l'impossibilité d'extension volontaire du membre et de l'état de paralysie permanente de celui-ci. L'existence de la branche aberrante du sciatique pourrait donner, selon les auteurs, la clé de l'étiologie de la parésie spasmodique qu'ils pensent avoir diagnostiquée chez le veau en question.

N.D.L.R. : Même remarque que pour [3!. 
5] Lauvergne J.-J., Blix P. C., ig68. Hereditary determinism of the cleft palate associated with ankylosis of limbs in Charolais cattle. XII Int. Conf. Génét., 1, 277.

Les résultats, déjà évoqués en [2], de la première campagne d'enquête parmi les veaux faisant l'objet d'un contrôle de descendance en race Charolaise pure, nés en 1967, sont repris, avec d'autres observations. Ils ne semblent pas contredire l'hypothèse selon laquelle la tare serait déterminée par un mutant récessif à pénétrance complète, à condition toutefois d'admettre que la fréquence du gène est très faible dans la population Charolaise femelle issue d'insémination.

[6] Logkay B., 1968a. La fissure palatine héréditaire associée à l'ankylose des membres en race Charolaise. $140 \mathrm{e}$ promotion, E.N.S.A., Grignon, i p. ronéoté.

Note préliminaire de :7?.

N.D.L.R. : 11 s'agit d'une contérence donnée à la chaire de \%ontechnie de l'École nationale supérieuse d'Agronomie de Grignon (Y'velines le 2.5.6 68).

[7] LogeAy B., I968b. Nouveaux résultats concernant la fissure palatine associée à l'ankylose des membres, anomalie héréditaire en race Charolaise. pp. $25+15$ tableaux +3 figures. ronéoté.

B. Logeay a suivi, dans l'Allier, la campagne de contrôle de descendance des veaux nés en I968 pour examiner les cas d'anomalies. La conclusion de son étude est que la tare est monofactorielle, récessive, à pénétrance totale ou subtotale. S'il y a dominance, en tout état de cause la pénétrance est faible chez l'hétérozygote.

Une analyse de l'évolution, chez les femelles, de la fréquence génique, semble cadrer avec l'hypothèse que cette fréquence aurait été nulle lorsqu'a commencé l'insémination artificielle dans l'Allier en 1952 , cependant que la fréquence chez les mâles avait déjà atteint la valeur d'équilibre actuelle.

Le rapport contient également les résultats d'une expérience de survie d'anormaux ainsi que l'analyse des pedigrees de 21 taureaux Charolais transmetteurs apparents de la tare. (On peut trouver au moins un ancêtre commun à tous 6 générations en arrière : CHAMPION HBC 7035 .

N.D.L.R. : Il s'agit du rapport d'un stage accompli par l'auteur au Département de Génétique animale de l'I.N.R.A., C.N.R.Z., Jouy en Josas, sous la direction de B. VISSAC et J.- J. LAuverg..e.

Le raisonnement de génétique des populations péche surtout par le fait qu'il est difficile d'admettre qu'au début de l'insémination artificielle dans l'Allier la fréquence du gène chez les femelles, non inscrites il est vrai mais de race pure, ait pu être nulle. L'usage de mâles inscrits parmi lesquels le gène était déjà répandu remonte en effet à très longtemps.

$$
1969
$$

8] Lauvergie J.-J., 1969. Étude de la fissure palatine en race Charolaise au C.I.A. de l'Allier, cámpagne 1969. Dpt de Génétique animale, C.N.R.Z., Jouy en Josas, pp. 3, ronéoté.

Peu avant le terme de la troisième campagne de testage, on constate que le pourcentage de taureaux transmetteurs apparents de la tare est assez constant d'année en année. On continue à admettre une récessivité à pénétrance totale mais on en vient à considérer que les variations d'expressivité de la tare peuvent englober des anomalies limitées aux membres, sans atteintes palatines.

9] Leipold H. W., Cates W. F., Radostits (). M., Howell W. E., 1969. Spinal dysraphism, arthrogryposis and cleft palate in newborn Charolais calves. Can. Vet. J., 10, 268-27.3.

Le premier cas examiné par un des auteurs remonte à 1965 . A partir de 1967 , après la création du Western College of Veterinary Medicine (W.C.V.M.) à Saskatoon, Saskatchewan, Canada, un certain nombre de veaux Charolais anomaliques ont été adressés, à des fins d'examen, à la clinique par des éleveurs du Saskatchewan et de l'Alberta, encouragés dans ce sens par la Canadian Charolais Association (C.C.A.). 
Douze de ces veaux nouveau-nés sont décrits ( $2 \delta^{\alpha}$, so $\left.q q\right)$. Huit d'entre eux avaient le palais fendu et les quatre membres atteints d'arthrogrypose; les quatre autres avaient seulement des atteintes des membres dont trois aux pattes avant uniquement. Les atteintes aux membres, toujours symétriques, variaient dans leur forme et leur intensité, de l'extrême flexion à l'extension avec une raideur plus ou moins grande ou, au contraire, une mobilité excessive.

Aucun des veaux atteints ne pouvait se tenir debout mais ils étaient normalement actifs. Les masses musculaires des membres atteints étaient de taille réduite et manquaient de tonus à la palpation. Une légère scoliose et une cyphose ont été parfois aussi observées. L'examen histologique a révélé des anomalies de la moelle épinière (sévère disraphisme) et des muscles des membres infirmes qui apparaissaient plus pâles et de taille réduite : peu de fibres normales qui étaient remplacées par du tissu adipeux. Ces défauts étaient d'autant plus marqués que les muscles étaient de taille plus réduite. Aucune étiologie n'est proposée pour ce syndrome.

[10, Lherminier P., I969. L'Analyse sanguine : conséquences zootechniques. Élevage et Insémination (I 12 ), I - 20.

Supposant que 25 taureaux Charolais mis à l'épreuve en France et transmetteurs apparents sont porteurs d'un gène responsable de la tare de fissure palatine, l'auteur avance, dans une courte note, qu'aucun des groupes sanguins $\mathrm{A}, \mathrm{B}, \mathrm{F}, \mathrm{J}, \mathrm{L}, \mathrm{M}, \mathrm{S}$ et $\mathrm{Z}$ ne présentent parmi ces mâles des caractéristiques différentes de celles de la population Chavolaise en général. Par ailleurs, les notes de pointage de 16 taureaux transmetteurs apparents ne semblent pas s'écarter de celles de la moyenne des taureaux de la race.

N.D.L.R. : L'analyse en question fait l'objet d'un chapitre intitulé " taureaux transmettant le caractère "fissure palatine ", p. I 5-I6.

\section{0}

[11; Howell W. E., Leipold H. W., 1970. An inherited defect in newborn Charolais calves. F.I.A.E.R.B.C., London, July 2, 1970, pp. $3+2$, ronéoté.

Première mention, devant les responsables Charolais du monde entier, de l'existence d'une anomalie congénitale caractérisée par des défauts du palais, des membres et de la colonne vertébrale. Les auteurs s'appuient sur l'analyse des pédigrees de veaux anormaux recueillis au W.C.V.M de Saskatoon (cf. [9]) pour avancer qu'il s'agit d'un défaut héréditaire, mendélien, monofactoriel. Ils pensent, en outre que les défauts des membres pourraient résulter du développement anormal d'une portion de la moelle épinière comprise entre les vertèbres cervicales $n^{\circ} 2$ et 5 .

Une liste des taureaux transmetteurs est donnée et les éléments d'une politique de détection et d'éradication de la tare sont proposés.

N.D.L.R. : Il s'agit d'un rapport présenté à l'Assemblée de la F.I.A.E.R.B.C. tenue à Londres en juillet 1970. Cet exposé très franc du problème causa à l'époque, un certain émoi dans le monde du Charolais. La mention "F.I.A.E.R.B.C., London" a été ajoutée dans le titre par la rédaction.

[1 2. J. M. D., r970. Réunion du 9 juin r970 du Groupe d'Étude clu caractère "Palais fendu en Charolais". I.T.E.B., Section "Amélioration génétique ", pp. 6 ronéoté.

Mis au courant par les soins du Département de Génétique de l'I.N.R.A., le Herd-Book Charolais (H.B.C.) et l'I.T.E.B., avaient demandé, au début de I970, que soit créé un groupe d'étude pour le problème posé par cette anomalie en race Charolaise avec les représentants des diverses organisations intéressées.

Le point des connaissances françaises acquises lors de l'enquête I.N.R.A.-I.T.E.B. sur les descendances des taureaux mis à l'épreuve a été fait lors de cette première réunion (cf. $[1],[2],[5],[6], 17],[8])$. En outre, pour la première fois il a été avancé que la pénétrance du facteur récessif responsable de l'anomalie pourrait être assez faible. Quant à l'éradication du gène l'accord se fait sur la nécessité de continuer la détection des transmetteurs lors de la mise à l'épreuve des taurillons sur leur descendance en race pure et de publier les résultats.

Les avis sont plus partagés quant à la pertinence de l'élimination de certains taureaux transmetteurs détectés. En effet, si la pénétrance est aussi faible qu'elle semble l'être, le maintien de certains hétérozygotes dans l'élevage, au moins en race pure, ne donnera guère plus d'anormaux que leur éradication. Par ailleurs, en n'utilisant que des mâles indemnes en I.A. on fera baisser la fréquence chez les femelles d'insémination rendant la détection future plus difficile. Toutefois, il est difficile, pour des raisons psychologiques et commerciales, au moins dans l'immédiat. d'admettre à l'agrément des taureaux transmetteurs avérés de la tare. 
N.D.L.R. : Les initiales J. M. D. désignent J.-M. Duplan, Directeur de la Section Amélioration Génétique de l'I.T.E.B. (anciennement C.T.C.D.) qui présidait la réunion.

[13] Kigffer N. W., Cartwright T. C., 1970. Inherited defects. Charolais Banner, Sept., I970, I 88-I 9 I.

Première mention, dans une revue biliographique consacrée aux défauts héréditaires du Charolais, de la tare dite "bent pastern cleft palate ". Cette analyse met à leur juste place, comme nous l'avons vu en 3, les travaux de GREELEY et al. (no 3 et 4 de la présente liste); elle se base également sur les travaux canadiens ( $\left.{ }^{\circ} 9\right)$, mais ignore encore les travaux français. Les auteurs pensent. mais sans apporter de preuves, que le défaut est héréditaire, mendélien, monofactoriel et récessif.

[I 4] LaUVergne J.-J., I 970 . Quelques considérations sur la fissure palatine en race Charolaise. pp. 2 , ronéoté.

Il s'agit de précisions pouvant aider à la discussion sur l'éradication abordée en I3. Avec une première estimation de la pénétrance chez les homozygotes égale à 0,20 , la fréquence génique étant estimée à $0, I_{5}$, on trouve quelques explications à la situation observée dans la race Charolaise et aux difficultés d'éradication quand les troupeaux sont de petite taille. Par ailleurs, on peut penser qu'une fréquence d'équilibre est atteinte et que des forces de sélection, qui pourraient avoir un effet économiquement intéressant, contrebalancent les pertes dues à l'apparition des anormaux.

N.D.L.R. : Supplément au compte rendu de la Réunion du 9 juin 70 (cote no 13 ). Ce topo, daté du 30 juin, a été diffusé plus tard.

i 5] Leipold H. W., Cates W. F., Radostits (). M., Howell W. E., 1970a. Arthrogryposis and associated defects in newborn calves. $.4 \mathrm{~m}$. J. Vet. Res., 31, I 367-74.

Six veaux Charolais anomaliques nouveaux dépéchés par les éleveurs au W.C.M.M. de Saskatoon ont été disséqués. Ils s'ajoutent aux cas décrits en [17] pour confirmeı lus ịrécédentes observations. Les auteurs se montrent encore moins conclusifs sur l'étiologie qu'en [9], d'autant plus que la présence du virus de la langue bleue a été détecté chez l'un des veaux tarés.

[i6] Leipold H. W., W. F. Cates, (). M. Rawostits, W. E. Howell. 197ob. Arthrogryposis... a bovine congenital defect Proc. an. meet. Can. Soc. anim. Prod. Ottawa July 5-9-1970, 2.5.

Résumé de [1 1 1 .

[17] Leipold H. W., Howell W. E., Radostits O. M., Cates W. F., 1970. Leg contracture and cleft palate in newborn calves. Can. Charolais Banner, July r970, 86-88.

Publié dans le journal officiel de la race Charolaise au Canada, cet article est destiné aux éleveurs, bien qu'il s'entoure d'une bibliographie assez importante. I'ne description détaillée et complète du syndrome est donnée, avec un appel à la coopération pour que de nouveaux cas soient acheminés vers le W.C.V.M. de Saskatoon où se font les dissections et les études du programme concerté canadien (cf. [9]).

Les auteurs sont moins définitifs qu'en [ I I ] en ce qui concerne l'étiologie qualifiée, ici, de possiblement héréditaire.

\section{I97I}

[I8] J. M D./C. B., 197I. Groupe d'étude du caractère "Palais fendu " en Charolais. Compte rendu du 13 octobre I971. I.T.E.B., Section Amélioration génétique, pp. 3, ronéoté.

Les conclusions du rapport i 8 sont, en gros, retenues par le groupe d'étude mais on demande qu'il soit procédé à des analyses caryotypiques afin que soit définitivement écartée l'hypothèse d'une anomalie chromosomique. 
N.D.L.R. : Les initiales J.M.D. désignent J.-M. Duplan qui présidait la réunion (cf. $\left.{ }_{[} \mathrm{I} 2\right]$ ). Des examens caryotypiques de deux veaux anormaux (un mâle et une femelle) ainsi que de leurs 4 parents normaux ont été effectués par P. Popescu du Laboratoire de Caryologie I.T.E.B.I.N.R.A. Tous les examens ont révélé des images normales de chromosomes. Les résultats n'ont pas encore été publiés.

[19] Lauvergne J.-J., I97I. Déterminisme héréditaire du Syndrome et conséquences pour la sélection du Charolais. Réunion du groupe d'étude "Palais fendu en Charolais" du I3 octobre 71. "Section Amélioration Génétique " de l'I.T.E.B., Paris, pp. 2, ronéoté.

Avec les résultats de cinq campagnes de contrôle de la descendance en Charolais pur, l'hypothèse avancée précédemment (en [ I 2] et [14]), qui faisait état d'une récessivité monofactorielle avec une pénétrance faible, semble se confirmer. Une estimation de la fréquence génique, faite en tenant compte de la non détection de certains transmetteurs et en supposant quel'équilibre de la fréquence génique est atteint dans les deux sexes, serait de 0,18 . Quant à la pénétrance chez les homozygotes, une estimée entre sexes pourrait être de 0,17 .

La fréquence du gène semble se maintenir grâce à un effet d'hétérosis qui s'exercerait plus probablement chez les femelles. Ainsi, malgré sa fréquence élevée ce gène d'anomalie ne cause dans le Charolais français que des pertes réduites $(0,5 \mathrm{p}$. I0o), et ces pertes pourraient. dans une certaine mesure, être compensées par un effet d'hétérosis.

20] Leipold H. W., Radostits O. M., Cates W. F., Howell W. E., Nawrot P., i97 I. Arthrogryposis and cleft palate in newborn Charolais calves. Canadian Charolais Association, 48I6 McLeod Trail, Calgary 6, Alberta, pp. 6 imprimé.

Tract rédigé par les chercheurs de Saskatoon et distribué aux éleveurs Charolais par la Canadian Charolais Association (C.C...A.) qui, depuis cette époque, a changé d'adresse cf. Annexe II.

N.D.L.R. : Sans date, daté d'après les clires des auteurs.

I 972

[21] Binns W., 1972. Syndrome de la déformation générale du squelette chez le veau. Le Charolais (25), 62-64.

Il s'agit d'une conférence prononcée à l'Assemblée Générale de la F.l.A.E.K.B.C. à Dallas, Texas, en octobre I97I.

L'auteur y expose l'étiologie d'anomalies congénitales qui ont, comme facteur commun, des déformations osseuses. Une enquête sur le terrain a, en particulier, prouvé avec certitude qu'en Amérique une anomalie congénitale associant des défauts articulaires des membres à la fissure palatine, pouvait être induite par la consommation d'une variété de lupin par les vaches gestantes.

N.D.L.R. : On a cru bon de citer cet article, bien qu'il ne traite pas exactement du sujet. L'existence d'une telle "mimique " ainsi présentée à tous les responsables de la race Charolaise a eu un effet stimulant sur les recherches concernant SAP dans la race Charolais'.

[22] Giroud A., I972. Syndromes arthro-myodysplasiques et leur cause chez l'homme et l'animal. Bull. Acad. Méd., 156, 8I 4-8I5.

Dans le cadre d'une revue des syndromes en France arthro-myodysplasiques, l'auteur signale qu'il a remarqué dans la zone Charolaise en France des veaux atteints d'anomalies articulaires frustres. Certains récupèrent l'usage normal de leurs membres par la suite. Aucune étiologie précise n'est avancée. 
[23] LAUVERGNe J.-J., 1972. Hereditary determinism of arthrogryposis with palatoschisis syndrome in Charolais cattle. Conférence donnée le 20 juin 1972 au Beef Cattle Research Ranch de l'Université d'Alberta à Kinsella, Alberta, non publiée.

Cette conférence présentait l'état d'avancement des travaux français du programme I.T.E.B.1.N.R.A. (cf. en particulier [1 2], [14], ] I8] et [19]). Avec les résultats de la dernière campagne, de nouvelles estimations de la fréquence génique dans la race $(0,20)$ et des pénétrances chez les homozygotes $\left(0, I_{4}\right.$ chez les mâles, o, Io chez les femelles) étaient données.

N.D.L.R. : C'est par cet exposé que les chercheurs canadiens d'Edmonton et de Saskatoon ont été informés des recherches françaises en cours. Cette conférence a été citée parfois sous le libellé donné en [24].

[24] Lalivergie J.-J., 1972-1973. Hereditary determinism of arthrogryposis with palatoschisis syndrome in Charolais cattle. To be published in A.G.S.A.

Forme sous laquelle [23] a été citée par certains auteurs canadiens, en [34], [37] et [39].

N.D.L.R.: Les initiales A.C.S.A. désignent les Annales de Génétique et de Sélection animale.

[25: Leipold H. W., Radostits O. M., Cates W. F., Howell W. E., Nawrot P., 1972. Arthrogryposis and cleft palate in newborn calves. Canadian Charolais Association, 4816 McLeod Trail, Calgary, Alberta, $\mathrm{T}_{2} 60 \mathrm{~A} 8, \mathrm{pp} .6$, imprimé.

Idem [20] avec des modifications de détail.

N.D.L.R. : Sans date, daté d'après les direş des auteurs.

[26j Singh I'. Ml., Little P. B., 1972. Arthrogryposis and cleft palate in a Charolais calf. Can. l.et. J., 13, $2 \mathrm{I}-24$.

Jusqu'à présent, les cas connus au Canada provenaient des provinces de l'Ouest, principalement Alberta et Saskatchewan; ici on a la première mention d'un cas apparu dans l'Ontario. Les descriptions anatomiques concordent assez bien, et les auteurs font un rapprochement entre des cavités observées dans la mœlle épinière et les anomalies des membres.

[27] Girol') A., Guteguex L., 1973. Sur les arthromyodysplasies chez le veau. Bull. Acad. Vit., 96, 443-446.

Détails sur les observations déjà évoquées en [2:2] de veaux arthromyodysplasiques (AMD) dans la zone Charolaise en France. Ces études sont confinées aux veaux AMD sans fente palatine. Leur fréquence serait de 1 à $2 \mathrm{p}$. Ioo. L'action de plantes vénéneuses et d'une carence en manganèse est à écarter. Plusieurs faits plaident en faveur du rôle de facteurs génétiques.

[28: (ikeene H. J., Leipold H. W., Huston K., GuFfy M. M., 1973. Bovine congenital defects: arthrogryposis and associated defects in calves. Am. J. liet. Res., 34, 887-891.

Sur I 122 veaux de toutes races atteints de malformations congénitales et examinés au College of I'eterinary Medicine de Manhattan (Kansas), I 88 étaient atteints d'arthrogrypose. ( 13 I des quatre membres et 57 de deux membres). Cent-vingt-deux présentaient en outre un. deux ou plusieurs défauts visibles annexes. Les défauts annexes étaient, le plus souvent, des défauts du squelette axial ou la palatoschisis. Malgré les similitudes apparentes les syndromes comportant l'arthrogrypose peuvent être classés en entités bien distinctes. Ainsi, en Charolais, le syndrome 
avec arthrogrypose et palatoschisis se différencie des syndromes similaires, spécialement de celui observé en Hereford. Les défauts du système nerveux central et des lésions musculaires existent chez l'un et non chez l'autre.

.29] Lauvergne J.-J., Lefort G., 1973. Nouvelle méthode pour analyser le comportement et la fréquence des gènes récessifs à effets visibles dans les populations bovines. $C . R$. Acad. Sci. Paris (Série D), 227, 2793-2796.

On a vu, en [19], qu'une fois admis le fait que l'anomalie était récessive à pénétrance incomplète il fallait, pour estimer la fréquence génique et la pénétrance, tenir compte de la non détection de certains taureaux transmetteurs potentiels, les groupes de descendants des taureaux de testage étant de taille finie. Il faut, en outre, tenir compte de la taille inégale de ces lots de descendants. En terme de génétique des populations il s'agit d'une analyse de demi-germains (les descendants d'un lot de testage étant demi-frères de père) pour laquelle il n'existait, jusqu'à présent, aucune solution mathématique. Une méthode d'estimation est proposée avec des hypothèses simplifiées correspondant en gros à la situation observée dans le testage des mâles Charolais en race pure pratiqué en France. Le système d'équation, qui se résoud par itération, donne une estimation de la fréquence du gène supposé récessif et de sa pénétrance chez les homozygotes des deux sexes.

\section{0] McLenna. W., 1973. Veterinary Notes. British Charolais Newsletter (7), 49.}

Brève mention, à l'usage des éleveurs britanniques de Charolais, de l'existence dans la race de l'anomalie des pattes et du palais.

.31] Nawrot P. S., 1973. Arthrogryposis, a congenital defect in newborn calves. M. S. Thesis, Dpt of Anim. Sci. Univ. Saskatchewan, Sept. 1973, pp. 138 , ronéoté.

Il s'agit d'une analyse des données recueillies à Saskatoon entre I 969 et 197 I dans le cadre du programme concerté canadien (cf. 9 et 12 ) : 79 cas. Une étude statistique des localisations anatomiques permet, tout d'abord, de dresser un profil de l'anomalie et de chiffrer les variations de son expressivité. L'examen en ferme des conditions de conduite des troupeaux, d'alimentation et de santé ne permet pas de dégager l'existence de causes exogènes. L'hypothèse de récessivité monofactorielle à pénétrance complète est alors testée sur des fratries de demi-germains (demifrères et sœurs de père) au sein desquelles est apparu un anomalique au moins et où le grand-père maternel est un mâle Charolais transmetteur avéré de la tare, la grand-mère maternelle étant une vache d'une autre race que l'on peut supposer, avec quelques raisons, être indemne de la tare. En éliminant de chaque fratrie le premier anormal apparu, l'auteur montre que la fréquence observée des anormaux ne s'écarte pas significativement de $r / 8$, proportion attendue dans le cas de monofactorialité autosomale récessive simple.

N.D.L.R. : On a là un bon exemple d'analyse de $3 / 4$ de germains, dans le cadre plus général de la méthode des indicateurs. Hustos et al. (1974) ont donné un aperçu des diverses variantes de la méthode des indicateurs (probands method) applicables aux anomalies héréditaires bovines.

\section{I974}

32] Anonyme, 1974a. Compte rendu du Comité Technique de la F.I.A.E.R.B.C., I 3 sept. 1974 Vichy, France, pp. $5+\mathrm{I}$, ronéoté.

La délégation canadienne qui s'exprime principalement par la voix du docteur TryPHonas (W.C.V.M., Saskatoon) voudrait que soient approfondis les travaux scientifiques sur l'arthrogrypose, spécialement les études morphologiques et enzymatiques. La délégation canadienne propose que cette recherche soit entreprise au W.C.V.M., elle demande pour cela une aide de la F.I.A.E.R.B.C. Le Comité Technique accepte cette proposition du Canada.

Un projet à l'étude dans son association est présenté par J.W. GosseTT, Secrétaire de l'A.I.C.A (American International Charolais Association). Ce projet, qui a pour objet de garantir avec une probabilité assez voisine de la certitude que tel ou tel reproducteur ne transmettra pas de tare 
récessive à sa descendance, n'est pas retenu par le Comité, en raison de son coût, des difficultés de sa. réalisation et des risques d'élimination de gènes favorables.

N.D.L.R. : En septembre 1974, les résultats déjà obtenus au Canada sont indéniables encore que sous estimés : le travail de Nawrot [3I] semble en effet peu connu, de même que celui de GOODEWARDENE [36] or ces deux thèses se recoupent et donnent, sans équivoque la clé de l'explication génétique. Fort de l'expérience acquise les canadiens du W.C.V.M. veulent aller plus loin mais cela va être plus ardu (vu le peu d'enzymes encore repérables la chance de tomber sur celle qui est reliée avec le phénomène reste faible) et plus coûteux (il va falloir créer un troupeau expérimental). L'aide financière de la C.C.A. qui n'a jusqu'alors pas été négligeable devra alors être renforcée, sinon relayée. Il semble normal aux Canadiens de faire appel à la communauté des éleveurs Charolais. A noter que c'est la première fois que le Comité Technique inscrit l'arthrogrypose à son ordre du jour.

[33] Axoxyme, r974b. Minutes of a Technical Meeting held on friday 13 th September r974, in Vichy, France, at 8.35 am, pp. 9, dactylographié.

11 s'agit d'un compte rendu sténographié de la réunion du Comité Technique de la F.I.A.E.R.B.C. coté 3 I dans la présente liste et qui complète ce document. Cette minute a été dressée à l'initiative de M. McKay, Secrétaire de la British Charolais Society (B.C.S.) et approuvé par le docteur McLexsax, Vétérinaire Consultant de cette association.

[34j Berg R. T., Goonewardene L. A., I974a. The genetics of Arthrogryposis in Charolais cattle. Ist World Congr. Genet. Appl. Anim. Breed., 1, 635-642.

Résumé de la thèse de Goonewardene [37] faite sous la direction du Professeur R. T. Berg.

[35] Berg R. T., Goonewardene L. A., I974b. Arthrogryposis or "crippled calf condition" in cattle. Univ. Alberta, Agr. Bull., Summer 74, 22-27.

Doublet, à usage de vulgarisation, de [34], en moins complet.

[36] B. H./A. T., 1974. A review of canadian Charolais breeders experience with arthrogryposis. pp. 2, ronéoté.

Lorsqu'elle a été mise en face du problème de l'anomalie articulaire et palatine, la Canadian Charolais Association (C.C.A.) a pris le parti de traiter ouvertement du problème, comme en témoigne le programme concerté mis en place avec l'Université du Saskatchewan. Elle a exhorté ses adhérents à signaler leurs anormaux et à les envoyer à Saskatoon (cf. déjà [9]). Par ailleurs la C.C.A. a réuni un troupeau femelles transmettrices avérées de la tare, ce qui a déjà permis de détecter des mâles porteurs. Cette politique n'est pas toujours facile à suivre. La collaboration des éleveurs n'est pas acquise a priori, et la C.C.A. doit agir avec précaution dans la diffusion du nom des taureaux porteurs, pour ne pas s'exposer à des actions en justice. Par ailleurs, le fait d'admettre l'existence d'une anomalie héréditaire en Charolais peut être utilisé par la concurrence pour freiner le développement commercial de la race.

N.D.L.R. : Les initiales B. H. sont celles de Bill HuNT, président de la C.C.A. Il s'agit d'un des deux rapports annexes à [32] présentés lors du Comité technique de la F.I.A.E. R.B.C. à Vichy.

[37: GoONewardene L. A., 1974. The genetics of arthrogryposis in Charolais cattle. M. S. thes., Dpt of Animal Science, University of Alberta, Edmonton. April 23, 1974, pp. 69, ronéoté.

tu Beef Cattle Research Ranch de l'Université de l'Alberta à Kinsella, Alberta, une souspopulation de transmetteurs reconnus ou suspectés de l'anomalie a été composée à partir d'une population métissée Angus, Charolais et Galloway créée en 196r. Chaque fois qu'une femelle a donné naissance à un anormal elle a été affectée à cette sous-population dite "troupeau estropié " (cripple herd), certains animaux suspects d'être porteurs comme étant fils et filles de parents porteurs. 
Au sein de ce " cripple herd " ont été effectués des croisements expérimentaux pour établir le mode d'hérédité de l'anomalie. Croisés entre eux les porteurs avérés de la tare donnaient une fréquence de $52 \mathrm{p}$. Ioo d'animaux, proportion significativement différente de $25 \mathrm{p}$. roo. L'écart provenait de la petite taille des fratries et de la possible existence d'homozygotes porteurs sains.

Une autre approche a consisté à considérer les veaux anormaux nés entre r970 et I973 de transmetteurs avérés et de transmetteurs suspects en tant que descendants de transmetteurs avérés. Chaque reproducteur était affecté d'une probabilité de transmettre le gène à sa descendance déterminée par son comportement comme porteur avéré ou son degré de parenté avec des porteurs avérés. On a pu ainsi établir que la probabilité moyenne d'apparition d'un anormal dans l'hypothèse de pénétrance totale en récessivité autosomale sur I3I croisements considérés était de 0,1185 . La fréquence observée a été de 0,145 , elle n'est pas significativement différente de la valeur calculée. L'auteur conclut que l'hypothèse de monofactorialité récessive avec pénétrance complète est acceptable.

La longévité et la fertilité de 20 femelles porteuses avérées a été comparée à celle de femelles témoins. Il est apparu que les femelles porteuses présumées hétérozygotes sont plus fertiles que les femelles témoins. Par ailleurs, ces mêmes femelles hétérozygotes, à âge égal, ont eu un taux de réforme mains élevé que les femelles de contrôle. L'auteur pense ainsi pouvoir expliquer, au moins partiellement, l'existence d'un équilibre de la fréquence génique qui pourrait s'établir dans les populations Charolaises et qui s'observerait déjà en France.

Comme la pénétrance totale ou subtotale observée au Canada l'est parmi des animaux qui 'n'ont qu'une certaine fraction de sang Charolais (la plupart du temps 3/4) et que tous les homozygotes porteurs sains décelés étaient des animaux de race pure, l'auteur pense que l'abaissement de la pénétrance en race pure peut être due à l'accumulation, par le jeu de la sélection naturelle, de modificateurs polygéniques. L'élimination complète du gène ne serait pas alors forcément économiquement souhaitable et même on pourrait utiliser en élevage certains de ses avantages.

[38] Lauvergne J.-J., I974. Étude des gènes à effets visibles à partir des données de testage des taureaux sur leur descendance. Ier Congr. Mond. Génét. Appl. Élev., 1, 47-55.

L'exposé de l'analyse des fratries de demi-germains donnée en [29] est brièvement reprise. 11 est ensuite montré comment on peut, par un raisonnement probabiliste, prouver que les anomalies diverses des membres et du palais, observées dans le Charolais en France sont rattachables à un même syndrome et que ce syndrome est héréditaire, mendélien, monofactoriel. Ce dernier test est basé sur des comparaisons à une loi de Poisson de la distribution des anormaux observés parmi les lots de descendants des taureaux de testage.

Par ailleurs, le comportement de la fréquence annuelle des mâles transmetteurs détectés dans le cadre du testage en race Charolaise pure en France, laisse à penser que la fréquence du gène a atteint une valeur d'équilibre dans la race. La plus simple explication d'un tel phénomène est l'hétérosis, comme on l'avait déjà avancé (cf. [I4] et [I9]).

\section{[39] L. Q./A. T., 1974. Arthrogryposis, the current state of knowledge.}

Une revue brève mais au fait des connaissances les plus avancées du moment. Bien que le maintien du gène dans la population n'apparaisse pas antiéconomique, même à une fréquence relativement haute, l'Association Charolaise Canadienne (C.C.A.) tient à contrôler son expansion et à en réduire sa fréquence en identifiant les transmetteurs possibles dans la population, par testage sur la descendance ou par tout autre moyen, que l'on demande aux chercheurs de trouver.

N.D.L.R. : Les initiales L. Q. sont celles de Lloyd QuAntz, secrétaire de la C.C.A. Il s'agit d'un des deux rapports annexes à [32] et présentés au Comité technique de la F.I.A.E.R.B.C. de Vichy.

[40] Anonyme, r975. Compte rendu des délibérations du Comité Technique de la F.I.A.E.R.B.C. réuni le 3 juillet 1975 à Calgary, Alberta, Canada. pp. 7, ronéoté.

Avec les arguments supplémentaires tirés des travaux français alors présentés (cf. [44]) le Comité se range à l'avis que les anomalies souvent associées du palais et des membres sont rattachables dans la race Charolaise à un syndrome monofactoriel mendélien que l'on pourrait désigner 
sous le nom de SAP (Syndrome d'Arthrogrypose et de Palatoschisis). En race pure, il est conseillé d'utiliser la mise à l'épreuve des taureaux sur leur descendance pour repérer, à moindre frais, les transmetteurs. La taille conseillée des lots de descendance est de 200.

Le programme de recherche canadien que la F.I.A.E.R.B.C. a envisagé de soutenir suite au rapport du Comité Technique de 1974 (cf. no $3 \mathrm{I}$ ) exposé par le docteur Tryphonas est en trois points :

I) création d'un troupeau expérimental pour reproduire par croisement l'anomalie, en vue de vérifier l'hypothèse génétique et d'inclure les différentes causes environnementales,

2) qualifier et quantifier aux plans cliniques, histopathologique et physiologique les différentes localisations de la tare,

3) rechercher les différentes possibilités d'association entre des marqueurs de nature variée (biologique, protéines, enzymes) et l'anomalie en cause.

Ce programme serait exécuté au W.C.V.M. de Saskatoon; il durerait 2 à 4 ans, son coût annuel serait de 30 ooo dollars. Le Comité va proposer au Conseil d'Administration de la F.I.A.E.R.B.C. son financement pour un an.

N.D.L.R. : Il y a également eu une version anglaise de ce compte rendu.

[4I] Faucon A., I975. Texte de l'intervention devant le Conseil d'Administration de la Fédération Internationale Charolaise, le 4 juillet 1975 à Calgary, pp. 2, ronéoté.

Les conclusions élaborées par le Comité Technique à Calgary (cf. 39) sont proposées au Conseil d'Administration de la F.I.A.E.R.B.C. par le Président du Comité technique.

[42] Grroud A., 1975. Les arthromyodysplasies dans l'espèce bovine. Rec. Méd. Vét., 151, $44 \mathrm{I}-446$.

Reprenant et complétant ses observations d'un précédent article (cote no 26 de la présente liste), l'auteur pense que, dans la race Charolaise, les atteintes frustres articulaires déjà décrites et qualifiées maintenant de " syndrome paturon " pourraient avoir la même étiologie que le cas plus net rattaché au syndrome qu'il appelle paturon-palais.

[43] Howell W. E., 1975. Neonatal defects of the bovine. In Research Report, Animal Science Research Coordinating Group. Univ. of Saskatoon : projects completed or in which there was significant progress. I p. ronéoté.

Bref résumé du travail de NAwRot (1973) (cote no. 29) qui avait été dirigé par le professeur W. E. Howell du Department of Animal Science University of Saskatchewan, Saskatoon.

N.D.L.R. : La partie interprétation génétique du programme concerté canadien Université de Saskatoon-C.C.A. est en effet assumé par les généticiens du Department of Animal Science de cette Université.

[44] LaUvergne J.-J., 1975a. État présent des connaissances sur le syndrome d'Arthrogrypose et de Palatoschisis (SAP) dans le bétail Charolais en France. Comité technique de la F.I.A.E.R.B.C., du 3 juillet 1975 , pp. I6, ronéoté.

Il s'agit d'un rapport invité fait à la demande des organisateurs de la réunion de la F.I.A.E.R.B.C. à Calgary, une première version de l'article paru un peu plus tard en français ([47]).

N.D.L.R. : L'estimation de la fréquence du gène dans la population française $(q=0,3 \mathrm{I})$ et celles des pénétrances chez les mâles homozygotes $\left(w_{m}\right)$ et chez les femelles homozygotes $\left(w_{f}\right)$ diffèrent quelque peu de celle de [47]. Elles ne doivent pas être retenues plus longtemps car il s'agissait d'une application approchée du système d'équation faite en un moment où, pressé par le temps, on n'avait pas eu la possibilité d'écrire et d'utiliser un programme sur ordinateur. 
[45] Lauvergne J.-J., 1975b. Present state of knowledge on the syndrome of Arthrogryposis and Palatoschisis (SAP) in french Charolais cattle. Technical comittee, World Federation of Chavolais, July $5^{\text {th }} 1975$, Calgary.

Idem [44] mais en anglais cette fois.

N.D.L.R.: En 43 ont donnée la date du 3 juillet et, en 44 celle du 5 . En fait le rapport a été présenté et discuté une première fois au Comité technique de la F.I.A.E.R.B.C. réuni le 3 juillet (cf. [40]) puis, de nouveau exposé en session pleinière de la F.I.A.E.R.B.C. le 5 comme article technique et distribué sous forme de tiré à part, dans les deux langues française et anglaise.

[46] Lauvergne J.-J., 1975c. SAP in canadian Charolais; some reflexions. pp. 6, ronéoté, diffusion restreinte.

A l'issue de la réunion de Calgary des propositions de recherche viennent compléter le projet canadien exposé en [40]. Il s'agirait d'éclaircir l'irritante question de la différence de pénétrance du gène en race pure et en croisement, d'estimer au Canada la fréquence actuelle du gène et son évolution possible, et de préciser la valeur du coefficient de sélection chez les hétérozygotes. Les données utilisées pourraient provenir, pour la plupart, d'enquêtes en fermes.

[47] Lauvergne J.-J., I975d. État actuel des connaissances sur le syndrome d'arthrogrypose et palatoschisis (SAP) dans le bétail Charolais de France. Ann. Génét. Sél. anim., 7. 321-330.

Les méthodes exposées en [38] ont été quelque peu améliorées, elles sont appliquées aux données de 7 campagnes de testage en race pure en France : 134 taureaux mis à l'épreuve, plus de I9 000 veaux nés, dont i ro SAP.

Tout d'abord, une analyse statistique des principaux symptômes observés sur les anormaux (fissure palatine, membres estropiés ou cyphose) montre que, pris individuellement, ces symptômes peuvent être considérés comme héréditaires. Des considérations sur leur association, sur un même individu et à l'intérieur de la descendance d'un même taureau, montrent qu'ils ne peuvent avoir chacun un déterminisme indépendant ni même provenir de facteurs linkés. On doit admettre, avec une probabilité proche de l'unité, qu'ils font partie d'un même syndrome héréditaire mendélien monofactoriel qui pourrait être appelé Syndrome d'Arthrogrypose et de Palatoschisis (SAP).

La constance de la fréquence des transmetteurs permet de penser que la fréquence génique est à l'équilibre depuis longtemps dans la population mâle et, par ricochet, dans la population femelle, vue l'ancienneté de l'insémination artificielle. Par ailleurs, on peut penser que le gène a un comportement uniquement récessif. Dans ces conditions, on peut appliquer les équations données en [29] pour l'estimation de la fréquence génique et des pénétrances chez les homozygotes.

Après passage sur ordinateur l'estimation de la fréquence génique a été de o,200 dans l'hypothèse basse (tous les cas douteux de SAP écartés) et de 0,202 dans l'hypothèse haute où l'on avait, au contraire conservé tous ces cas.

Les 'estimations des pénétrances étaient de o, I I 5 (hypothèse basse) et de $0, I_{3}$ I (hypothèse haute) entre sexes. Ces estimations diffèrent peu de celles données en I972 (cf. [23]).

Pour le moment en France on ne peut encore préciser à quel niveau intervient l'effet d'hétérosis qui semble devoir être postulé pour expliquer l'équilibre de la fréquence génique observé. Une certaine politique d'éradication surtout pour les taureaux de testage est d'ores et déjà mise en pratique mais dans les troupeaux privés toutefois, les structures de l'élevage français sont telles qu'en tout état de cause l'éradication, même systématique, ne peut avoir qu'une portée limitée.

[48] Quavrz L., x975. Business is good. Can. Charolais Banner, 9 (6), 50.

Au sein d'un article qui traite de questions diverses il y a un bref avis, à l'usage des adhérents du C.C.A. Ceux qui désirent mettre au testage pour SAP tel taureau leur appartenant sont priés de s'inscrire, le troupeau femelle de transmettrices connues a été agrandi. 
[49] Quantz L., 1975. C.C.A. newsletter. Can. Charolais Banner, 9 (7), 78.

Rappel descriptif du dispositif mis en place depuis quelques années déjà par la C.C.A. en liaison avec le W.C.V.M. : identification des veaux SAP et leur acheminement vers un laboratoire d'analyse, identification des femelles et des mâles transmetteurs de la tare, publicité faite aux renseignements obtenus et leur utilisation.

\section{ANALYSE SUCCINCTE ET DISCUSSION}

Les sources imprimées ou ronéotées relatives à SAP peuvent se ranger selon leur origine (Canada, France, États-Unis, Grande-Bretagne, organismes internationaux) et leur genre (articles originaux dans des revues scientifiques ou assimilées ; article de vulgarisation dans revue technique ou tract, rapports à diffusion restreinte ou compte rendu de réunion).

En faisant abstraction de 5 doublets, 3 triplets et I quadruplet, on peut classer les $3^{8}$ cotes restantes selon leur origine et leur genre (tabl. I).

\section{TABLEAU I}

Ventilation des cotes des sources imprimées et ronéotées concernant SAP, selon leur genre et leur origine

Repartition of the references concerning SAP according their type and their origin

\begin{tabular}{|c|c|c|c|c|c|c|c|}
\hline 1 & Canada & France & $\begin{array}{l}\text { États- } \\
\text { Unis }\end{array}$ & $\begin{array}{c}\text { Grande- } \\
\text { Bretagne }\end{array}$ & $\begin{array}{l}\text { Organismes } \\
\text { inter- } \\
\text { nationaux }\end{array}$ & $\begin{array}{c}\text { Effectifs } \\
\text { lignes }\end{array}$ & $\begin{array}{l}\text { Pour- } \\
\text { centage }\end{array}$ \\
\hline $\begin{array}{c}\text { Articles originaux } \\
\text { dans revues scientifiques } \\
\text { ou assimilées }\end{array}$ & $\begin{array}{l}{[9],[15]} \\
{[17],[26]} \\
{[31],[37]} \\
(6)\end{array}$ & $\left\{\begin{array}{c}{[1],[5],[10],} \\
{[22],[27],} \\
{[29],[38],} \\
{[42],[47](9)}\end{array}\right.$ & $\begin{array}{l}{[3],[4]} \\
{[13],[21]} \\
\text { (4) }\end{array}$ & 1 & & 19 & 50,0 \\
\hline $\begin{array}{c}\text { Articles de vulgarisation } \\
\text { dans revues techniques } \\
\text { ou tracts }\end{array}$ & $\begin{array}{c}{[10],[48]} \\
{[49]} \\
(3)\end{array}$ & & $\begin{array}{l}{[28]} \\
(1)\end{array}$ & $\begin{array}{l}{[30]} \\
(1)\end{array}$ & & 5 & 13,2 \\
\hline $\begin{array}{c}\text { Rapport } \\
\text { à diffusion restreinte } \\
\text { ou comptes rendus } \\
\text { de réunion }\end{array}$ & $\begin{array}{l}{[11],[36],} \\
{[39],[45],} \\
\text { (4) }\end{array}$ & $\begin{array}{c}{[2],[7],[8]} \\
{[12],[14]} \\
{[18],[19]} \\
(7)\end{array}$ & & & $\begin{array}{c}{[32],[10]} \\
{\left[\begin{array}{l}4 \\
1\end{array}\right]} \\
(3)\end{array}$ & 14 & 36,8 \\
\hline Effectifs colonnes & 13 & 16 & 5 & 1 & 3 & 38 & \\
\hline Pourcentage & 34,2 & 42,1 & 13,2 & 2,6 & 7,9 & & \\
\hline
\end{tabular}

Cette ventilation a exigé que l'on fasse certains choix, qui peuvent paraître arbitraires, notamment pour la référence conservée quand il y a doublet ou triplet : on a retenu celle qui était classable dans le genre le plus scientifique. Le choix lui-même des genres contient aussi une part d'arbitraire, de même que la recherche des doublets et des triplets. Pratiquement 
il n'y a pas deux articles exactement semblables, sauf des versions dans deux langues différentes, et encore. En outre, même très voisins, deux articles, conçus pour toucher des publics divers, ont incontestablement leur utilité. Par ailleurs des articles apparemment très différents peuvent n'être, sous l'angle qui nous intéresse, que des répétitions. Le choix que nous avons fait est donc forcément dans plusieurs cas un compromis.

Ceci dit on voit se dégager un certain nombre de tenclances et de faits. Tout d'abord apparait l'importance, déjà soulignée dans l'introduction, de la part prise par les rapports à diffusions restreintes et les comptes rendus de réunion ( $37 \mathrm{p}$. roo des cotes). Dans le cas de la France par exemple ces rapports ont, entre 1968 et $\mathbf{1 9 7 5}$, été le seul support des résultats de l'enquête I.N.R.A.I.T.E.B. Au Canada, si cette fâcheuse tendance à retarder les publications scientifiques a été moins nette, il n'en reste pas moins que les 2 travaux académiques bien documentés et conclusifs de Nawrot [3I] et de Goonewardene [37] n'ont pas encore été publiés d'une manière suffisamment détaillée dans des périodiques scientifiques.

A la décharge des auteurs négligents, il faut dire que l'on peut hésiter devant la présentation de résultats pour lesquels on attend encore quelques confirmations. Il serait cependant toujours possible de donner des notes initiales pour prendre date et renseigner les laboratoires travaillant parallèlement. Quant aux thèses, elles devraient donner lieu à des mémoires détaillés qui d'habitude se font attendre.

En ce qui concerne, maintenant, les origines des sources on note la dominance, avec une importance à peu près semblable, des travaux français et canadiens (resp : $34 \mathrm{p}$. Ioo et $42 \mathrm{p}$. Ioo des cotes). Le léger avantage numérique de la France est probablement dû au fait que nous n'avons pas pu inventorier les minutes des comités techniques plus ou moins formels qui se sont nécessairement tenus au Canada. Si on met en parallèle les recherches françaises et canadiennes on doit noter tout d'abord une certaine similitude dans la date du commencement (1965-1966), dans les associations qui se sont créées entre une unité de recherche d'une part et un organisme technique d'autre part (W.C.V.M.-C.C.A. au Canada, I.N.R.A.-I.T.E.B. en France). La similitude n'est toutefois pas totale car même si elle est maintenant coordonnée avec le reste des travaux canadiens le programme de l'Université d'Edmonton avec l'utilisation d'un troupeau expérimental est assez original et indépendant de l'action concertée C.C.A.-W.C.V.M. Le second projet français, animé par le professeur Giroud (cotes [2I], [26] et [4I]), correspondait quant à lui à une étude beaucoup plus ponctuelle. En outre, l'action concertée française est essentiellement une analyse de génétique de population de données recueillies en ferme alors que les dominantes au Canada sont la typologie de l'anomalie et l'analyse de croisements expérimentaux et de pédigrees. Il y a bien eu en France, au départ, des études anatomiques en liaison avec la Chaire d'Anatomie. de l'École Vétérinaire d'Alfort, mais elles n'ont pas été développées, en l'absence d'un système de récupération des anomaliques que, pour des raisons matérielles, on n'a pu mettre en place.

En fait, les recherches dans l'un des deux pays ne pouvait pas démarquer exactement celles dans l'autre car le comportement du gène diffère sensiblement selon que l'on se trouve en race pure (en France) ou en croisement (au Canada), avec au maximum $7 / 8$ de sang Charolais. Dans le premier cas, la pénétrance très faible a obligé à une approche statistique plus élaborée alors que, dans le second cas, les formules habituelles de l'analyse des indicateurs suffisaient. Par une sorte de hasard il s'est trouvé que la qualité et le nombre des données disponibles convenaient exactement aux deux situations. En France l'existence de très nombreux lots de descendants de mâles choisis au hasard et accouplés à des femelles pure race elles aussi choisies au hasard permettait l'estimation à la fois de la pénétrance et de la fréquence génétique, alors qu'au Canada on disposait de données plus classiques de $3 / 4$ de germains (même père et même grand-père maternel transmetteur avéré de la tare (HowELL-NAwROT, Saskatoon) ou bien des individus dont il était possible aesez aisément (la pénétrance étant totale) de déterminer leur probabilité de porter la tare (BERG-Goonewardene, Edmonton).

On manque encore de détails sur la liaison entre la pénétrance et le pourcentage de sang Charolais - on sait seulement que la pénétrance est totale ou subtotale lorsque le pourcentage est au plus de $7 / 8$ de sang (résultats canadiens) - alors qu'elle est assez basse en race pure : au 
maximum o, 4 (résultat français). On aimerait bien pouvoir suivre cette évolution chez les animaux présentant $7 / 8,15 / 16$ et $31 / 32$ de sang Charolais tels qu'ils existent au Canada.

A part cette divergence quant à la pénétrance, les résultats obtenus des deux côtés de l'Atlantique se recoupent et se complètent assez bien. Lorsque les Canadiens d'Edmonton ou de Saskatoon ont testé l'hypothèse groupée : déterminisme monofactoriel autosomal, récessif, à pénétrance incomplète avec la méthode des indicateurs il leur fallait admettre que tous les anormaux croisés Charolais atteints aux pattes et/ou au palais présentaient bien le même syndrome, ce qui a été prouvé en France.

Les calculs français, quant à eux, reposaient sur le fait qu'un équilibre de la fréquence génique a été atteint. Or, pour que cet équilibre soit il faut qu'existe, quelque part, un effet d'hétérosis, ce que précisément les Canadiens semblent avoir prouvé, à partir des observations faites à Kinsella par les chercheurs d'Edmonton.

\title{
CONCLUSION
}

Par chance les études jusqu'à présent pratiquement non coordonnées se sont avérées complémentaires. Cette complémentarité subsistera d'autant mieux que l'information circulera plus vite et plus totalement entre responsables de l'élevage et scientifiques.

Rę̧u pour publication en janvier 1976.

\section{SUMMARY}

\author{
THE SYNDROME OF ARTHROGRYPOSIS AND PALATOSCHISIS (SAP) \\ IN CHAROLAIS CATTLE, AN ANNOTATED BIBLIOGRAPHY : I967-I975
}

During the period 1967 to 1975 there appeared in the literature 49 papers by 33 different authors dealving with the syndrome of Arthrogryposis and Palatoschisis (SAP) in the Charolais breed. It was difficult to annotate a large proportion of these papers $(37 \mathrm{p}$. Ioo) in the usual documentary method because of the need to condense the very long articles (theses) whereas others were very concise formal reports of meetings. The Canadian ( 34 p. roo) and French ( 42 p. roo) reports concern investigations initiated at about the same time (r966) as collaborations between research establishments and breed organisations. The detailed results of the Canadian and French work are in sufficient agreement as to leave no doubt that SAP is a typical monofactorial autosomal recemie trait unique to the Charolais breed. There is evidence of a difference in penetrance values - low in pure French Charolais but almost 100 p. 100 in the case of defectives carrying $7 / 8$ Charolais breeding in Canada but this requires more precise investigation. The frequency of the gene in the pure Charolais in France is o.20. During the past two years there has been some international cooperation in the planning and funding of research on SAP whereas prior to that the exchange of information was less than desirable.

\section{RÉFÉRENCES BIBLIOGRAPHIQUES}

Huston K., Chase R., Waller R., r974. Identifying genes with visible effects in cattle : full, threequarter and half-sib; other methods. Ist World Congr. genet. appl. anim. Breed., Madrid, 1, 39-46.

Lauvergne J.-J., Boyazogle J. G., Hubert D., x968. Le phénomène culard chez les bovins. Bibliographie annotée. Bull. tech. Dép. Génét. anim. (I.N.R.A., Fr.) (2), pp. 49.

LAUVERGNE J.-J., r972. Utilisation consciente et inconsciente de certains facteurs mendéliens dans la sélection des grosses es pèces animales. Ann. Génét. Sél. anim., 4, 79-85. 


\section{ANNEXE I}

\section{Abréviations utilisées dans le texte et dans l'annexe II (')}

A.I.C.A. : American international Charolais Association (Houston, Texas).

B.C.C.S. : British Charolais Cattle Association (Leamington Spa, Great Britain).

C.C.A. : Canadian Charolais Association (Calgary, Canada).

C.T.C.D. : Centre technique pour le Contrôle de la Descendance, une branche de l'I.T.E.B. devenue Section d'Amélioration génétique (Paris, France).

F.I.A.E.R.B.C. : Fédération Internationale des Associations d'Éleveurs de la race bovine Charolaise (Nevers, France).

H.B.C. : Herd-Book Charolais (Nevers, France).

I.N.R.A. : Institut National de la Recherche Agronomique (France) auquel appartient le Département de Génétique animale localisé au C.N.R.Z., Jouy en Josas.

I.T.E.B. : Institut Technique de l'Élevage Bovins (Paris, France).

S.A.P. : Syndrome d'Arthrogrypose et de Palatoschisis.

W.C.V.M. : Western College of Veterinary Medicine (University of Saskatchewan, Saskatoon, Sask., Canada).

\section{ANNEXE II}

Index des auteurs cités avec leur qualité et leur adresse $\left(^{2}\right)$

BERG R. T.

Binns W.

BLIN P. C.

Boyd C. L.
Dr, Professor, Department of Animal Science, University of Alberta, Edmonton, Alberta $\mathrm{T} 6 \mathrm{G} 2 \mathrm{E}_{3}$, Canada.

\section{[33], [34]}

[20] of Agriculture, Logan, Utah, U.S.A.

Dr, Professeur, Chaire d'Anatomie, École nationale vétérinaire, 9470I Alfort, France.

Dr, Associate Professor, Department of Veterinary Medicine and Surgery, College of Veterinary Medicine, Texas A and $M$ University, College Station, Texas, 77843 , U.S.A.

Cartwright T. C. Dr, Professor, Animal Science Department, Texas A and M University, College Station, Texas 77843 , U.S.A.

Cates W. F. Dr, Department of Veterinary Clinical Studies, W.C.V.M., University of Saskatchewan, Saskatoon, Sask., S7NOWO, Canada.

Duplan J. M. Directeur de la Section d'Amélioration Génétique de l'I.T.E.B., 149, rue de Bercy, 75579 Paris Cedex I2, France.

FAucon A. Directeur-Adjoint de l'I.T.E.B., I49, rue de Bercy, 75579 Paris Cedex I2, France.
$[15], \quad[167$ [19], [24]

[I3], [I7]

[40] tion.

(1) Les abréviations sont également décryptées dans le texte, au moins lors de leur première utilisa-

(2) Telles qu'elles sont connues de nous fin 1975 . 
Giroud A. Dr, Professeur Honoraire d'Embryologie, Faculté de Médecine de Paris, rue des Saints-Pères, 75006 Paris et Chemilly, ozz ro Souvigny, France.

Goonewardene L. A. Dr, Department of Animal Science, University of Alberta, Edmonton, Alberta T6G $2 \mathrm{E}_{3}$, Canada.

$[21],[26],[4 I]$

$[33],[34 !,[36]$

Gossett J. W. Secretary, A.I.C.A., I6ro, Old Spanish Trail, Houston, Texas 77025, U.S.A.

Greeley R. G. Dr, Associate Professeur, Department of Veterinary Anatomy, College of Veterinary Medicine, Texas A and M Iniversity, College Station, Texas $77^{8} 43$ U.S.A.

$[3 \mathrm{I}]$

$[3],[4]$

Grense H. J. Dr, College of Veterinary Medicine, Kansas State Iniversity, Manhattan, Kan. 66506, U.S.A.

Gúfguex I.. Dr, Dept de Nutrition, Centre National de Recherches zootechniques (I.N.R.A.), $7835^{\circ}$ Jouy en Josas, France.

(;UfFy M. M. I)r, College of Veterinary Medicine, Kansas State University, Manhattan, Kan. 66506, U.S.A.

Howell. W. F. Ir, Professor, Animal Science Department, University of Saskatchewan, Saskatoon, Sask. S7NOWO, Canada.

Hunt B., Former President CC.A Endiang, Alberta Canada.

Hustox $\mathbf{k}$. Dr, Director of the Minnesota Agricultural Experiment Station, Coffey Hall, University of Minnesota, Saint-Paul, Minn. 65I01, U.S.A.

JOLLY.D. G. Adresse inconnue.

Kieffer N. M. Dr, Professor, Animal Science Department, Texas A and M University, College Station, Texas 77843, U.S.A.

LAuvergxe J.-J.Dr, Département de Génétique animale, Centre national de Recherches zootechniques (I.N.R.A.), $7835^{\circ}$ Jouy en Josas, France.

LEFORT (i. Professeur, Chaire de Mathématique, Institut national Agronomique, 16, rue Claude Bernard, 75005 Paris, France.

LeIPold H. W. Dr, Professor, College of Veterinary Medicine, University of Kansas, Manhattan, Kan. 66506, U.S.A.

Lhermixitr P. Ingénieur, Section Amélioration Génétique, I.T.E.B. I 49, rue de Bercy, 75579, Paris, Cedex 12, France.

$[27]$

$[26]$

[27]

[9], [ 12$],[15],[16]$.

[19], [24], [42]

[35]

[27!

[3], [t]

[I I ]

[I], [2:, [5], [8],

[14], [18], [22],

[23], [28], [37],

$[43],[44],[45]$,

$[46$ ?

[28]

[9], [1 2], [1 5], [16],

[19], [24], [27]

[10]

Little P. B. Dr, Department of Pathology, Ontario V'eterinary College, Guelph, Ontario, Canada.

LogEAy $B$. Adresse inconnue.

McKAy A. Secretary BCCS, 6 Coventry Road, Cubbington, Leamington Spa CV32 7JN, Warwickshire, Great Britain.

McLesiax W. Dr, Veterínary Field Station, Easter Busch, Roslin, Midlothian, Great Britain.

Nawrot P. I)r, Nat'l Inst. Environmental Health Sci., bldy 17 , Room 1706 P.O. Box 12233. Research Triangle Park, North Carolina, 27709 , USA.

Quaxtz I. (ieneral Manager, CCA, 218 ith Av. SW Calgary, Alberta, T2 ROC 3 , Canada.

Radostits (). M. I)r, Professor, Department of Veterinary Clinical Studies, W.C.V.M., University of Saskatchewan, Saskatoon, Sask. $\mathrm{S}_{7} \mathrm{NOWO}$, Canada.

Sirvgh I'. M. Dr, Central Animal Husbantry Station, Kluang, Johore, Malaysia

Tryphoxas I. I)r, Heath and Welfare Canada. Toxicology Research Divi;ion, Tunney's Pasture, ()ttawa, (Ontario, Ki Aol _2, Canada.

$[25]$

$[6],[7]$

[32]

โ29i, [32!

[19], [24], [30]

[38], [47], [48]

$[9],[15],[16],[19]$,

[24]

[25]

[31], [39] 


\section{ANNEXE III}

\section{Bibliographie alphabétique par noms d'auteurs (1)}

[32] Anonye, r974a. Compte rendu du Comité technique de la F.I.A.E.R.B.C., I 3 sept. 1974, Vichy, France, pp $5+\mathrm{r}$, ronéoté.

[33] Anonyme, r974 $b$. Minutes of a technical Meeting held on friday 13 th september 1974 , in Vichy, France, at 8.35 pm, pp. 9 (dactylographié).

[40] Anonyme, 1975. Compte rendu des délibérations du Comité technique de la F.I.A.E.R.B.C. réuni le 3 juillet 1975 à Calgary, Alberta, Canada, pp. 7, ronéoté.

[34] Berg R. T., Goonewardene L. A., r974a. The genetics of Arthrogryposis in Charolais cattle. 1st World congr. Genet. appl. anim. Breed., 1, 635-642.

[35] Berg R. T., Goonewardene L. A., I974 b. Arthrogryposis or " crippled calf condition " in cattle. C'niv. Alberta Agr. Bull., Summer 74, 22-27.

[36] B. H./A. T., 1974. A review of Canadian Charolais breeders experience with arthrogryposis, pp. 2, ronéoté.

[2I] Binns W., 1972. Syndrome de la déformation générale du squelette chez le veau. l.e Charolais (25), 62-64.

[4I] Faucon A., I975. Texte de l'intervention devant le Conseil d'Administration de la Fédération internationale charolaise, le 4 juillet 1975 à Calgary, pp. 2, ronéoté.

[22] Giroud A., I972. Syndromes arthro-myodysphasiques et leur cause chez l'homme et l'animal. Bull. Acad. Méd., 156, 814-815.

[27] Giroud A., Guéguen L., 1973. Sur les Arthromyodysplasies chez le veaur. Bull. Acad. Vét., 96, $443-446$.

[37] GoONEWARDENE L. A., 1974. The genetics of arthrogryposis in Charolais cattle. M. S. thes. Dpt. of Animal Science, University of Alberta, Edmonton, April 23, 1974, pp. 69, ronéoté.

[3] Greeley R. G., Boyd C. L., Jolly D. G., I968. Bovine anomalies and spastic paresis. Southwest Vet., 12, 277-280.

[4] Greeley R. G., Jolly D. (i., 1968. A clue to the etiology of spastic paresis. Southwest Vet., 12, 189-191.

[28] Greene H. J., Leipold H. W., Huston K., Guffy M. M., I973. Bovine congenital defects : Arthrogryposis and associated defects in calves. Am. J. Vet. Res., 35, 887-89r.

[43] Howeli W. E., 1975. Neonatal defects of the bovine. In Research Report, Animal Science Research coordinating Group, Univ. of Saskatoon: Projects completed or in which there was significant progress. I p., ronéoté.

[iI] Howell W. E., Leipold H. W., 1970. An inherited defect in newborn Charolais calves. F.I.A.E.R.B.C. London July 2, 1970, p. $3+2$, ronéoté.

[12] J. M. D., I97o. Réunion du 9 juin r97o du Groupe d'Étude du caractère "Palais fendu en Charolais". ITEB, Section Amélioration génétique, pp. 6, ronéoté.

[18] J. M. D./C. B., 1971. Groupe d'Étude du caractère "Palais fendu " en Charolais. Compte rendu du I3 octobre I97I. ITEB Section Amélioration génétique, pp. 3, ronéoté.

[13] Kieffer N. M., Cartwright T. C., I970. Inherited defects. Charolais Banner, sept. 1970, I88-rgr.

[2] Lauvergne J.-J., 1967. Brève analyse des premiers résultats de l'enquête sur la fissure palatine en race Charolaise au C.I.A. de l'Allier, pp. $6+3$, ronéoté.

[8] Lauvergne J.-J., rg69. Étude de la fissure palatine en race Charolaise au C.I.A. de l'Allier, campagne r969. Dpt. de génétique animale, C.N.R.Z., Jouy en Josas, pp. 3, ronéoté.

[14] LAUvergne J.-J., r97o. Quelques considérations sur la fissure palatine en race Charolaise, pp. 2, ronéoté.

[19] LaUvergne J.-J., 1971. Déterminisme héréditaire du syndrome et conséquences pour la sélection du Charolais. Réunion du Groupe d'Étude "Palais fendu en Charolais » du 13 octobre $197 \mathrm{I}, \mathrm{pp}$. 2, ronéoté.

[23] Lauvergne J.-J., 1972. Hereditary determinisme of Arthrogryposis with palatoschisis Syndrome in Charolais cattle. Conférence donnée le 20 juin 1972 au Beef Cattle Research Ranch de l' ('niversité d'Alberta à Kinsella, Alberta, non publiée.

[24] LaUvergne J.-J., I972-r973. Hereditary determinism of arthrogryposis with palatoschisis syndrome in Charolais cattle, to be published in AGSA never published.

[38] Lauvergne J.-J., I974. Étude des gènes à effets visibles à partir des données de testage des taureaux sur leur descendance. 1 1 ${ }^{\text {er }}$ Congr. Mond. Génét. appl. Elev., 1, 47-55.

(1) Les numéros entre crochets [ ] renvoient à la bibliographie annotée. 
[44] Latvergne J.-J., I975a. État présent des connaissances sur le Syndrome d'Arthrogrypose et de Palatoschisis (SAP) dans le bétail Charolais en France. Comité technique de la F.I.A.E.R.B.C. du 3 juillet 1975 , pp. I6, ronéoté.

[45] Latvergne J.-J., I975 b. Present state of knowledge on the syndrome of Arthrogryposis and Palatoschisis (SAP) in french Charolais cattle. Technical Committe, World Federation of Charolais, July 5 th, r975 Calgary.

[46] Latvergne J.-J., r975C. État actuel des connaissances sur le Syndrome d'Arthrogrypose et de Palatoschisis (SAP) dans le bétail Charolais de France. Ann. Génét. Sél. anim., 7, 321-330.

[r] Lacvergne J.-J., Blin P. C., r967. Fissure palatine héréditaire associée à l'ankylose des membres dans la race Charolaise. Ann. Zootech., 16, 291-300.

[5] Lauvergne J.-J., Blin P. C., 1968. Hereditary determinisms of the cleft palate associated with ankylosis of limbs in Charolais cattle. $X I I^{\mathrm{e}}$ Int. Conf. Génét., 1, 277.

[29] Lauvergne J.-J., Lefort G., 1973. Nouvelle méthode pour analyser le comportement et la fréquence des gènes récessifs à effets visibles dans les populations bovines. $C$. $R$. Acad.Sci. Paris (série D), 277, 2793-2796

[9] Leipold H. W., Cates W. F., Radostits O. M., Howell W. E., rg69. Spinal dysraphism arthrogryposis and cleft palate in newborn Charolais calves. Can. vet. J., 10, 268-273.

[15] Leipold H. W., Cates W. F., Radostits O. M., Howell W. E., r970a. Arthrogryposis and associated defects in newborn calves. Am. J. Vet. Res., 31, r367-74.

[i6] Leipold H. W., Cates W. F., Radostits O. M., Howell W. E., 1970 $b$. Arthrogryposis a bovine congenital defect. Proc. Can. meet. can. soc. anim. Prod., Ottawa, July 7-9 1970, 25.

[17] Leipold H. W., Howell W. E., Radostits O. M., Cates W. F., I97o. I.eg contracture and cleft palate in newborn calves. Can. Charolais Banner, July 1970, 86-88.

[20] Leipold H. W., Radostits O. M., Cates W. F., Howell W. E., Nawrot P., I97i. Arthrogryposis and cleft palate in newborn Charolais calves. Canadian Charolais Association, 4816 MCLeod Trail, Calgary 6, Alberta, pp. 6 imprimé.

[25] Leipold H. W., Radostits O. M., Cates W. F., Howell W. E., Nawrot P., I972. Arthrogryposis and cleft palate in newborn calves. Can. Charolais Assoc., 4816 McLeod Trail, Calgary Alberta, T2GOA8, pp. 6, imprimé.

[1 Io] Lherminier P., r969. L'analyse sanguine : conséquences zootechniques. Élevage et insémination (I 12$), \mathrm{I}-20$.

[6] Logear B., I968. La fissure palatine héréditaire associée à l'ankylose des membres en race Charolaise. $140^{\mathrm{e}}$ promotion, E.N.S.A. Grignon, I p. ronéoté.

[7] Logeay B., 1968 b. Nouveaux résultats concernant la fissure palatine associée à l'ankylose des membres, anomalie héréditaire en race Charolaise ronéoté p. $25+15$ tabl. + figures.

[39] L. Q./A. T., 1974. Arthrogryposis, the current state of knowledge, pp. 3, ronéoté.

[30] McLennan W., 1973. Veterinary notes British Charolais Newsletter (7), 49.

[3I] Nawrot P.S., 1973. Arthrogryposis a congenital defect in newborn calves. M.S. Thesis, dpt. of Animal Sci. Univ. Saskatchewan. Sept. 1973, pp. 138, ronéoté.

[48] Quantz L., 1975. C.C.A. Newsletter. Can. Charolais Banner, 9 (7), 78.

[49] Quantz L., 1975. Business is good. Can. Charolais Banner, 9 (6), 50.

[26] Singh U. M., Lirtle P. B., 1972. Arthrogryposis and cleft palate on a Charolais calf. Can. Iet. J., 18, 2 I-24. 